\title{
Development and validation of a bedside instrument to predict carbapenem resistance among gram-negative pathogens in complicated urinary tract infections
}

\author{
Marya D. Zilberberg MD, MPH ${ }^{1,2}$, Brian H. Nathanson $\mathrm{PhD}^{3}$, Kate Sulham $\mathrm{MPH}^{4,5}$, Weihong Fan $\mathrm{MS}^{4}$ and \\ Andrew F. Shorr MD, MPH \\ ${ }^{1}$ EviMed Research Group, Goshen, Massachusetts, ${ }^{2}$ Universtiy of Massachusetts, Amherst, Massachusetts, ${ }^{3}$ OptiStatim, Longmeadow, Massachusetts, \\ ${ }^{4}$ The Medicines Company, Parsippany, New Jersey, ${ }^{5}$ Melinta Therapeutics, Lincolnshire, Illinois and ${ }^{6}$ Washington Hospital Center, Washington, DC
}

\begin{abstract}
We developed a bedside instrument to predict carbapenem resistance in complicated urinary tract infections. A model assigning weighted points for admission from an extended care facility (1), history of weight loss (1), early mechanical ventilation (1), age $<50$ years (2), male gender (3), catheter-associated urinary tract infection (4), prior antibiotics treatment (4), and prior carbapenem-resistant infection (8) exhibited good discrimination (C statistic, 0.721).
\end{abstract}

(Received 26 March 2018; accepted 8 June 2018; electronically published July 31, 2018)

Carbapenem resistance has arisen with alarming speed. ${ }^{1,2}$ Currently, $>3 \%$ of Enterobacteriaceae are carbapenem nonsusceptible, and rates among Pseudomonas aeruginosa and Acinetobacter baumannii are substantially higher, making it difficult for clinicians to select initially appropriate empiric coverage for patients presenting with serious infections. ${ }^{3,4}$ Although rapid diagnostic testing promises to improve early antimicrobial therapy targeting, it presents certain limitations and cannot be utilized without some assessment of pretest probability for resistance. ${ }^{5}$ A predictive tool based on clinical factors for risk stratification is necessary either as an adjunct to or a substitute for rapid diagnostic testing.

\section{Methods}

We developed a bedside score to predict the risk for carbapenem resistance within a multicenter retrospective cohort of hospitalized patients with complicated urinary tract infection (cUTI) diagnosis and a culture positive for $P$. aeruginosa, A. baumannii, Stenotrophomonas maltophilia (assumed always to be carbapenem resistant), or Enterobacteriaceae (see Supplemental Material). Because this study used fully deidentified retrospective data, it was exempt from institutional review board review.

Adult patients with a cUTI fitting our enrollment criteria were included (see Supplemental Material for enrollment criteria and

Author for correspondence: Marya Zilberberg, MD, MPH, PO Box 303, Goshen, MA 01032. E-mail: evimedgroup@gmail.com

Cite this article: Zilberberg MD, et al. (2018). Development and validation of a bedside instrument to predict carbapenem resistance among gram-negative pathogens in complicated urinary tract infections. Infection Control \& Hospital Epidemiology 2018, 39, 1112-1114. doi: 10.1017/ice.2018.166 identification algorithms). The data for the study were extracted from the Premier Research database, an electronic laboratory, pharmacy, and billing data repository, for 2009-2016 (through the third quarter of 2006), which contains $\sim 15 \%$ of all hospitalizations nationwide. (A detailed description of the database is available in Zilberberg et al. ${ }^{3,4}$ ) We used data from 178 US institutions submitting microbiology data into the database.

Carbapenem resistance was defined as a cultured organism designated as intermediate or resistant to imipenem, meropenem, ertapenem, or doripenem. A carbapenem-susceptible organism was an organism susceptible to either any carbapenem tested or to any third-generation cephalosporin (see Supplemental Material). First detection of a carbapenem-resistant organism (or a carbapenem-susceptible organism of interest in the absence of carbapenem resistance) served as the index culture. Empiric antibiotic treatment was considered appropriate when coverage within 2 days of the culture being obtained included the corresponding organism. All other regimens were deemed inappropriate empiric treatment. A cUTI was classified as community onset if it was present on admission or if an index culture was obtained within the first 2 hospital days.

\section{Statistical analyses}

We compared the carbapenem-resistant group to the carbapenemsusceptible group with respect to demographics, comorbidities, hospital characteristics and processes, and outcomes. To develop a logistic regression to predict the presence of carbapenem resistance, we used a split-cohort method, with $60 \%$ allocated to the training cohort and $40 \%$ to the validation cohort. We then pared the model to 10 predictors or fewer for the ease of the end user. Terms were 
culled based on the factor's prevalence and its contribution to the model's predictive ability in the development cohort using a stepwise bootstrapping algorithm and Bayesian information criterion in a series of nested models. ${ }^{6}$ We assessed the model's discrimination with the $\mathrm{C}$ statistic and calibration visually in a plot of observed versus expected risk deciles and with the Hosmer-Lemeshow statistic. We then developed a score considering the weight of each factor's regression coefficient to predict the presence of a carbapenem-resistant organism.

\section{Results}

Among 25,285 patients with cUTI, 23,469 patients (92.8\%) had a community-onset UTI and 1,357 (5.4\%) had a UTI caused by a carbapenem-resistant organism. Baseline characteristics, microbiology, and hospital events can be found in the Supplemental Material Tables 5-7.

Unadjusted mortality $(3.7 \%$ vs $2.3 \% ; P<.001)$ and 30 -day readmission $(24.3 \%$ vs $18.0 \% ; P<.001)$ were higher in the setting of carbapenem resistance than carbapenem susceptibility. Unadjusted median hospital costs were also greater among patients with carbapenem-resistant infections those with carbapenemsusceptible infections: $\$ 10,356$ (interquartile range [IQR], $\$ 6,509-\$ 18,674$ versus $\$ 8,613$ (IQR, $\$ 5,241-\$ 15,316$ ), respectively $(P<.001)$. Postinfection-onset hospital lengths of stay were also longer among patients with carbapenem-resistant infections than those with carbapenem-susceptible organisms: 5 days (IQR, 3-9 days) versus 4 days (IQR, $2-7$ days), respectively $(P<.001)$.

In a model designed to predict the likelihood of carbapenem resistance in this population, we assigned weighted points proportional to the regression coefficients of the following factors: admission from an extended-care facility (1), history of weight loss (1), early mechanical ventilation (1), age $<50$ (2), male gender (3), catheter-associated UTI (4), prior antibiotics treatment (4), and prior carbapenem-resistant infection (8). The model exhibited good discrimination in both the training ( $\mathrm{C}$ statistic, 0.746) and validation ( $\mathrm{C}$ statistic, 0.721$)$ sets. It performed better among hospitals with lower prevalence of carbapenem resistance, as evidenced by a C-statistic drop with an increase in carbapenem resistance prevalence in the validation set: tertile $1,0.752$ (prevalence of carbapenem resistance, 2.1\%); tertile 2, 0.725 (prevalence of carbapenem resistance, $4.7 \%$ ); and tertile 3, 0.703 (prevalence of carbapenem resistance, 9.2\%).

The distribution of patients within specific score ranges with the corresponding proportions of carbapenem resistance is shown in Fig. 1. While most of the cohort (86.5\%) fell under 10 points, nearly $15 \%$ of those patients with the score of 10 points or more harbored a carbapenem-resistant organism. Conversely, patients whose score was below 3, which accounted for $29.9 \%$ of all the patients in the cohort, had a $\sim 1 \%$ risk of a cUTI caused by a carbapenem-resistant organism.

\section{Discussion}

In a cohort of hospitalized patients with a cUTI ( $>5 \%$ carbapenem resistant), we developed a bedside predictive score that, by virtue of its high negative predictive value in the low score ranges, may be useful in helping clinicians rule out the need for empiric coverage for a carbapenem-resistant organism in a cUTI.

Although we did not limit our cohort to community-onset infections, the vast majority of the admissions (>92\%) had cUTI

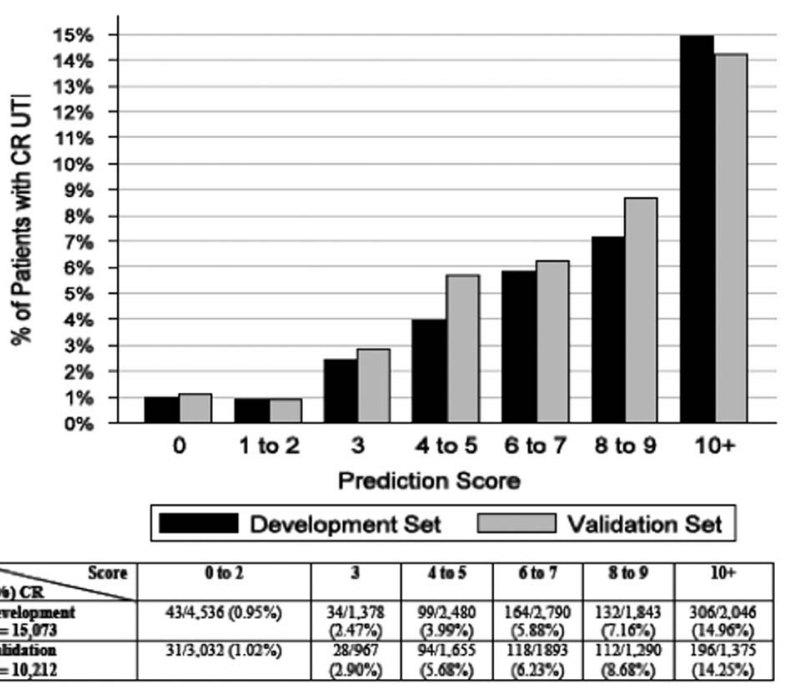

Fig. 1. Prediction score for carbapenem resistance in CUTI. Note. cUTI, complicated urinary tract infection; $\mathrm{CR}$, carbapenem resistant.

present on hospital day 1 . The small fraction of cUTIs that occurred as a nosocomial complication reflects well on hospital infection control interventions aimed at preventing such complications but also highlights the importance of considering the risk of a carbapenem-resistant organism even among patients from a community setting. Other investigators have noted this phenomenon in multiple pathogen classes, and we confirm its importance. $^{7-10}$

Previous investigations have demonstrated that the presence of resistance alone does not determine the outcome., ${ }^{3,4}$ Rather, whether the patient receives appropriate initial antibiotic regimen has a greater effect. Our score, though aiming to aid with such risk stratification, does not have perfect precision. However, when combined with knowledge of local resistance patterns and hospital antibiograms, it should facilitate efforts to ensure that patients with cUTIs receive appropriate empiric treatment. Moreover, our risk score helps to reinforce efforts toward antimicrobial stewardship by identifying when coverage for carbapenem-resistant pathogens in this syndrome is clearly not appropriate. Therefore, if applied correctly, this tool can help limit indiscriminate use of certain broad-spectrum antimicrobials.

Our study has a number of strengths and limitations. As a large multicenter cohort it is representative of US institutions, and thus has broad generalizability. Although our findings are susceptible to selection bias, we mitigated it by setting a priori enrollment criteria and definitions. Though some misclassification is possible, our cUTI algorithm was designed to maximize the specificity of the diagnosis, and carbapenem resistance conformed to a standard definition.

In summary, the bulk of cUTI admissions are community onset, and carbapenem-resistant pathogens account for $>5 \%$ of all gram-negative organisms at risk for developing carbapenem resistance. Until rapid diagnostics are ready for widespread use, we may need to settle for imperfect but helpful probabilistic models like ours to help identify, if not patients at high risk for resistance, those whose low probability of a resistant organism combined with other aspects of the clinical picture makes narrower-spectrum agents an adequate first choice for treatment.

Supplementary material. To view supplementary material for this article, please visit https://doi.org/10.1017/ice.2018.166 
Acknowledgments. Although Ms Sulham and Ms Fan are employees of the sponsors and participated in the study as coinvestigators, the larger sponsors had no role in study design, data analysis or interpretation or publication decisions. The data from this study have been presented as an oral presentation at the 28th European Congress of Clinical Microbiology and Infectious Diseases (ECCMID) 2018 meeting.

Financial support. This study was funded by The Medicines Company, Parsippany, New Jersey, and by Melinta Therapeutics, New Haven, Connecticut.

Conflicts of interest. Dr Zilberberg is a consultant to The Medicines Company and Melinta Therapeutics. Her employer, EviMed Research Group, has received research grant support from The Medicines Company and Melinta Therapeutics. Dr Nathanson's employer, OptiStatim, has received support from EviMed Research Group. Ms Sulham was an employee of and stockholder in The Medicines Company and is currently an employee of Melinta Therapeutics. Ms Fan is an employee of and stockholder in The Medicines Company. Dr Shorr is a consultant to and has received research grant support from The Medicines Company and Melinta Therapeutics. Drs Zilberberg and Shorr have received grant support and/or have served as consultants to Merck, Tetraphase, Pfizer, Astellas, Shionogi, and Theravance.

\section{References}

1. Centers for Disease Control and Prevention (CDC). Vital signs: carbapenemresistant enterobacteriaceae. Morb Mortal Wkly Rep 2013;62:165-170.

2. Braykov NP, Eber MR, Klein EY, Morgan DJ, Laxminarayan R. Trends in resistance to carbapenems and third-generation cephalosporins among clinical isolates of Klebsiella pneumoniae in the United States, 1999-2010. Infect Control Hosp Epidemiol 2013;34:259-268.

3. Zilberberg MD, Nathanson BH, Sulham K, Fan W, Shorr AF. Multidrug resistance, inappropriate empiric therapy, and hospital mortality in Acinetobacter baumannii pneumonia and sepsis. Crit Care 2016;20:221.

4. Zilberberg MD, Nathanson BH, Sulham K, Fan W, Shorr AF. Carbapenem resistance, inappropriate empiric treatment and outcomes among patients hospitalized with Enterobacteriaceae urinary tract infection, pneumonia and sepsis. BMC Infect Dis 2017;17:279.

5. Zilberberg MD, Shorr AF. Impact of prior probabilities of MRSA as an infectious agent on the accuracy of the emerging molecular diagnostic tests: a model simulation. BMJ Open 2012;18:2(6). doi: 10.1136/bmjopen-2012001804.

6. Austin PC, Tu JV. Bootstrap methods for developing predictive models. Am Statistician 2004;58:131-137.

7. Guh AY, Bulens SN, Mu Y, et al. Epidemiology of carbapenem-resistant Enterobacteriaceae in 7 US communities, 2012-2013. JAMA 2015; 314:1479-1487.

8. Tang HJ, Hsieh CF, Chang PC, et al. Clinical significance of communityand healthcare-acquired carbapenem-resistant Enterobacteriaceae isolates. PLoS One 2016;11(3):e0151897.

9. Doi Y, Park YS, Rivera JI, et al. Community-associated extendedspectrum $\beta$-lactamase-producing Escherichia coli infection in the United States. Clin Infect Dis 2013;56:641-648.

10. Pitout JD, Nordmann P, Laupland KB, Poirel L. Emergence of Enterobacteriaceae producing extended-spectrum $\beta$-lactamases (ESBLs) in the community. J Antimicrob Chemother 2005;56:52-59. 